\title{
Modelling the optical and radiative properties of freshly emitted light absorbing carbon within an atmospheric chemical transport model
}

\author{
M. Kahnert \\ Swedish Meteorological and Hydrological Institute, 60176 Norrköping, Sweden \\ Received: 16 October 2009 - Published in Atmos. Chem. Phys. Discuss.: 26 November 2009 \\ Revised: 26 January 2010 - Accepted: 2 February 2010 - Published: 8 February 2010
}

\begin{abstract}
Light absorbing carbon (LAC) aerosols have a complex, fractal-like aggregate structure. Their optical and radiative properties are notoriously difficult to model, and approximate methods may introduce large errors both in the interpretation of aerosol remote sensing observations, and in quantifying the direct radiative forcing effect of LAC. In this paper a numerically exact method for solving Maxwell's equations is employed for computing the optical properties of freshly emitted, externally mixed LAC aggregates. The computations are performed at wavelengths of $440 \mathrm{~nm}$ and $870 \mathrm{~nm}$, and they cover the entire size range relevant for modelling these kinds of aerosols. The method for solving the electromagnetic scattering and absorption problem for aggregates proves to be sufficiently stable and fast to make accurate multiple-band computations of LAC optical properties feasible. The results from the electromagnetic computations are processed such that they can readily be integrated into a chemical transport model (CTM), which is a prerequisite for constructing robust observation operators for chemical data assimilation of aerosol optical observations. A case study is performed, in which results obtained with the coupled optics/CTM model are employed as input to detailed radiative transfer computations at a polluted European location. It is found that the still popular homogeneous sphere approximation significantly underestimates the radiative forcing at top of atmosphere as compared to the results obtained with the aggregate model. Notably, the LAC forcing effect predicted with the aggregate model is less than that one obtains by assuming a prescribed mass absorption cross section for LAC.
\end{abstract}

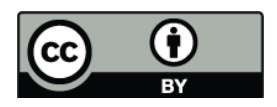

Correspondence to: M. Kahnert (michael.kahnert@smhi.se)

\section{Introduction}

Coupling atmospheric chemical transport models (CTM) to models for aerosol optical properties (AOP) is a challenging task with high relevance in chemical data assimilation and climate research. One major problem in chemical data assimilation of aerosol remote sensing observations is the construction of aerosol observation operators (Kahnert, 2008). An observation operator is a forward model that maps the output of the CTM to observed quantities, such as aerosol optical depth, or aerosol backscattering and extinction coefficients. This forward model is employed in a data assimilation algorithm to solve the inverse problem of retrieving aerosol physical and chemical properties from remote AOP observations. Another important application within climate modelling is the computation of the direct radiative forcing effect of aerosols. General circulation models (GCM) have often employed climatological aerosol fields. However, the trend in modern earth-system modelling is to treat aerosol fields dynamically by coupling a CTM to a GCM. To make use of the information provided by a CTM in calculations of the direct radiative forcing effect of aerosols, it is essential to have an accurate and robust method for mapping the aerosols' physical and chemical properties to their radiative properties.

By far the simplest and most wide-spread method for computing AOP based on size-resolved chemical composition is to approximate aerosols by homogeneous spheres. The different chemical aerosol components are, in general, assumed to be partially externally, partially internally mixed. The AOP are computed within the homogeneous-spheres approximation (HSA) by use of effective medium theory (Chýlek et al., 2000) and by Lorenz-Mie theory (Mie, 1908). This

Published by Copernicus Publications on behalf of the European Geosciences Union. 
approach is applicable for many liquid-phase water-soluble aerosols. However, it is well known that for non-spherical mineral aerosols the optical properties (Mishchenko et al., 1997; Nousiainen et al., 2006; Kahnert and Nousiainen, 2006; Veihelmann et al., 2006; Kahnert, 2004; Schulz et al., 1999, 1998) and even the climate forcing effect of aerosols (Pilinis and Li, 1998; Jacobson, 2000; Kahnert and Kylling, 2004; Kahnert et al., 2005, 2007; Otto et al., 2009) can be highly morphology-dependent. In such cases, more sophisticated optical models need to be employed (Mishchenko et al., 1996; Dubovik et al., 2002; Rother et al., 2006).

Mineral dust is one class of aerosol particles for which the HSA usually fails. Another class of aerosols with complex morphologies are light absorbing carbonaceous aggregates, commonly referred to as "soot", "black carbon" (BC), or "light absorbing carbon" (LAC). Freshly emitted LAC can be regarded as hydrophobic, externally mixed agglomerates of primary spherules. As these particles age, they are oxidized in the atmosphere, become partially hydrophillic, and can mix internally with other weakly absorbing aerosols. Uncertainties in modelling optical properties of LAC aggregates arise mainly from (i) variability in the refractive index; and (ii) approximations in the treatment of particle morphology. This article will focus on freshly emitted, externally mixed LAC aggregates, and on how to treat the complex morphology of such particles in AOP/CTM computations. Problems specific to internally mixed LAC are, e.g., discussed by Fuller (1999); Bond et al. (2006); Kocifaj and Videen (2008); Worringen et al. (2008).

For AOP computations of fractal-like aggregates, approximate methods, such as Rayleigh-Debeye-Gans (RDG) theory (Sorensen, 2001), and other approximations (Okada et al., 2007) have been developed and applied, e.g. by Zhao and Ma (2009). In parallel, methods based on rigorous electromagnetic scattering theory have been developed (Borghese et al., 1984; Mackowski and Mishchenko, 1996; Xu and Gustafson, 2001) and applied to various case studies. Applications to atmospheric LAC have, e.g., been reported by Liu et al. (2008). Astrophysical applications to interplanetary dust aggregates have, e.g., been presented by Okada et al. (2008).

In an excellent recent review paper, Bond and Bergstrom (2006) discussed the state-of-the-art of different methods and concluded that the use of the HSA for fresh LAC is likely to introduce significant errors in modelling AOP based on size and chemical composition of aerosols. Although the RDG approximation can be expected to give improved results, it still under-predicts LAC-absorption by about $30 \%$. As methods based on rigorous theory have, so far, proved to be computationally too demanding for broadband applications, the authors recommended for externally mixed LAC to (i) use a mass absorption cross section (MAC) of $(7.5 \pm 1.2) \mathrm{m}^{2} / \mathrm{g}$ at a wavelength of $\lambda=550 \mathrm{~nm}$, where the mass density of LAC can be assumed to be $1.8 \mathrm{~g} / \mathrm{cm}^{3}$; (ii) assume a single scattering albedo of $\omega=0.25$; (iii) assume an inverse wavelength dependence of MAC.
In this paper, a method for computing the scattering and absorption properties of fresh LAC based on rigorous electromagnetic theory will be employed (Xu and Gustafson, 2001). This method has been developed by astrophysicists, who are mainly interested in light scattering by interplanetary dust, which usually is much less absorbing than LAC. The focus in the present study is on assessing the feasibility of coupling this method to CTM computations with the idea of using it for developing aerosol optical observation operators for chemical data assimilation, and for performing radiative flux computations for constraining the direct climate forcing effect of LAC. The results are compared to corresponding computations based on the HSA and on prescribed MAC.

Section 2 briefly reviews some of the main physical properties of freshly emitted LAC. Section 3 explains the methodology employed in this study. Results are presented in Sect. 4, and discussed in Sect. 5.

\section{Physical properties of freshly emitted light absorbing carbon}

\subsection{Morphological properties}

Light absorbing carbon aggregates formed in combustion processes typically have a fractal-like structure that can be modelled by the scaling relation (Jones, 2006)

$N_{\mathrm{s}}=k_{0}\left(\frac{R_{\mathrm{g}}}{a}\right)^{D_{\mathrm{f}}}$,

where $N_{\mathrm{s}}$ denotes the number of spherical monomers in the aggregate, $a$ denotes the monomere radius, $k_{0}$ and $D_{\mathrm{f}}$ are known as the structure factor and the fractal dimension, respectively, and $R_{\mathrm{g}}$ is the radius of gyration of the aggregate, which is defined by

$R_{\mathrm{g}}=\sqrt{\frac{1}{N_{\mathrm{s}}} \sum_{i=1}^{N_{\mathrm{s}}} r_{i}^{2}}$.

Here $r_{i}$ denotes the distance of the $i$ th sphere in the aggregate from the centre of mass. Following Mackowski (2006) and Sorensen and Roberts (1997), we assume $D_{\mathrm{f}}=1.82$ and $k_{0}=1.27$, which correspond to typical values of freshly emitted LAC formed in diffusion limited cluster aggregation processes. The monomer radius typically ranges between 10 and $25 \mathrm{~nm}$ (Bond and Bergstrom, 2006). In this study, we shall assume $a=25 \mathrm{~nm}$. We used the aggregation algorithm by Mackowski (1994) to generate random fractal geometries. Figure 1 shows an example for $N_{\mathrm{s}}=1000$.

As this article focuses on freshly emitted LAC, it will be assumed throughout this study that the LAC aggregates are hydrophobic and not internally mixed with other chemical aerosol components. Note, however, that the hygroscopic and morphological properties of LAC change as the aggregates age in the atmosphere. The aggregates become more 


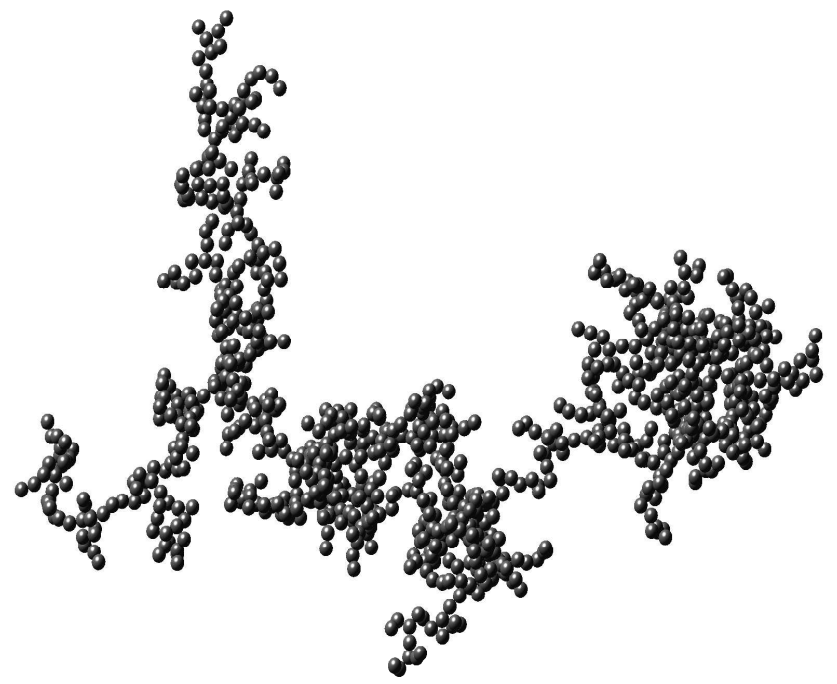

Fig. 1. Light absorbing carbon aggregate with $N_{\mathrm{S}}=1000$ primary monomers, fractal dimension $D_{\mathrm{f}}=1.82$, and structure factor $k_{0}=1.27$.

hydrophillic, and the fractal dimension increases to values $D_{\mathrm{f}}>2$. Aged LAC aggregates tend to mix with other aerosols components, such as sulphate aerosols (Worringen et al., 2008).

\subsection{Size range}

We need to obtain a first very rough estimate of the size range we have to consider in our optical modelling study. Figure 2 (solid line) shows a schematic number size distribution $n(r)$ of diesel soot emissions based on Färnlund et al. (2001). The majority of particles is emitted in the size range up to a radius of $100 \mathrm{~nm}$. In fact, some aerosol dynamic models assume that all externally mixed LAC is limited to the Aitken mode, such as the M7 model (Vignati et al., 2004), thus neglecting externally mixed LAC in the accumulation mode. However, since the optical cross sections increase with particle size, even small number densities of large particles can make a significant contribution to the size-averaged optical cross sections. Let us consider the extinction cross section $C_{\text {ext }}(r)$ of homogeneous spheres with a refractive index of $m=1.70+0.64 i$ (typical for LAC at $440 \mathrm{~nm}$ ), and compute $C_{\text {ext }}(r) n(r)$. The result is represented by the dashed line in Fig. 2. Particles up to a radius of approximately $r=300 \mathrm{~nm}$ can make a significant contribution to the total extinction efficiency.

Measurement methods of LAC size distributions, such as those employed by Färnlund et al. (2001), are often based on aerodynamic size segregation methods. Thus, the radius in Fig. 2 is to be understood as the radius of an aerodynamically equivalent sphere, denoted by $R_{\mathrm{a}}$. This quantity is difficult to convert into physically more tangible size measures, such as the radius of gyration $R_{\mathrm{g}}$, the radius $R_{\mathrm{A}}$ of a sphere

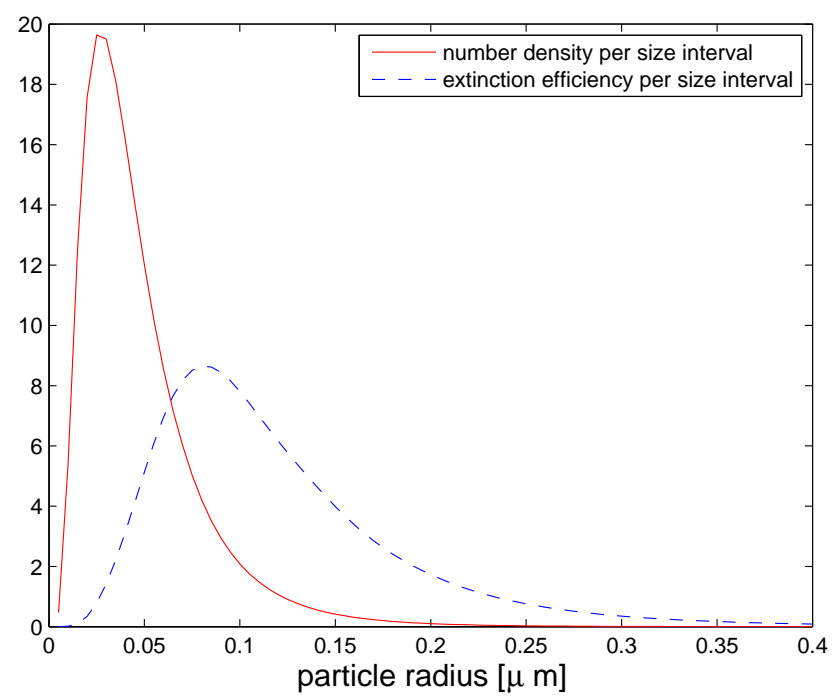

Fig. 2. Diesel soot emissions (solid line), and corresponding extinction efficiency per size interval (dashed line) for homogeneous spheres with a refractive index of $m=1.70+0.64 i$.

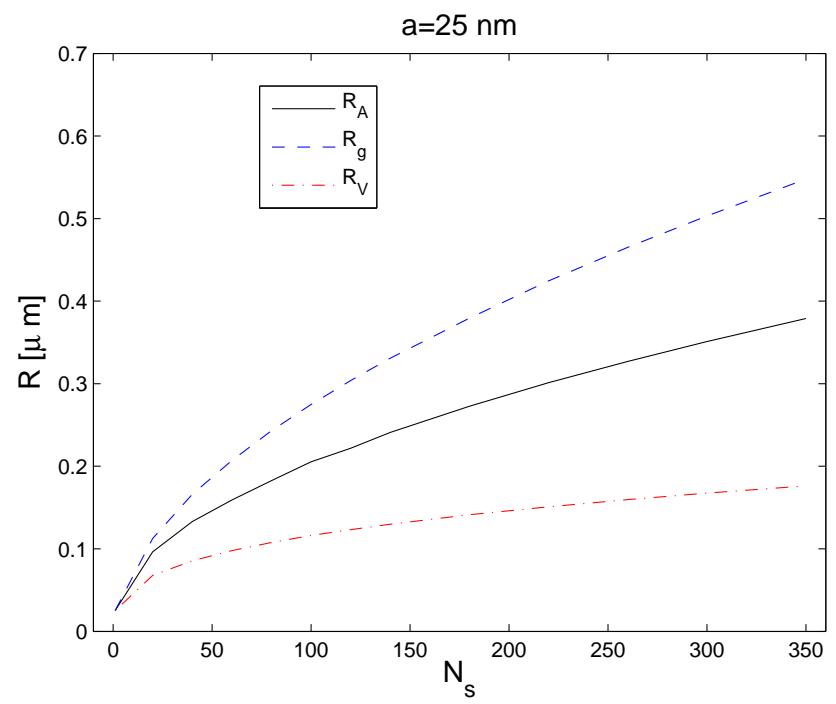

Fig. 3. $R_{\mathrm{A}}, R_{\mathrm{g}}$, and $R_{\mathrm{V}}$ as a function of $N_{\mathrm{s}}$ for aggregates with $a=25 \mathrm{~nm}, D_{\mathrm{f}}=1.82$, and $k_{0}=1.27$.

with equivalent geometric cross section, or the radius $R_{\mathrm{V}}$ of a sphere with equivalent volume. However, it can be assumed that for fractal clusters $R_{\mathrm{a}}$ lies much closer to $R_{\mathrm{A}}$ than to $R_{\mathrm{V}}$ (Smekens et al., 1997). Figure 3 shows $R_{\mathrm{A}}, R_{\mathrm{g}}$, and $R_{\mathrm{V}}$ as a function of the number of primary monomers $N_{\mathrm{S}}$ for the aggregates considered in this study. A size of $R_{\mathrm{A}}=300 \mathrm{~nm}$ would correspond to about $N_{\mathrm{s}}=250$. Since this is only a very rough estimate, we extend this range by a factor of 4 up to aggregates sizes of $N_{\mathrm{s}}=1000$. This should safely cover the entire size range (in terms of mass per particle) that can be reasonably expected to contribute to the extinction cross 
section. Note, however, that for smaller monomer radii $a$, aggregates may even be larger than $N_{\mathrm{s}}=1000$.

Since we are interested in coupling AOP and CTM simulations, and since CTMs are primarily mass transport models, the size of the LAC aggregates shall from now on be specified in terms of $R_{\mathrm{V}}$.

\subsection{Refractive index}

The refractive index $m=n+i \kappa$ of LAC can vary depending on the degree of graphitisation and depending on the void fractions in the material. These two effects give rise to different inter-dependencies of the real part $n$ and imaginary part $\kappa$ of $m$.

In the graphitisation model the refractive index of LAC is explained in terms of the molecular structure of the carbonaceous material, as discussed in detail by Bond and Bergstrom (2006). LAC usually consists of a mixture of carbon bonds known as $s p^{2}$ and $s p^{3}$ bonds. In the former, one $s$ and two $p$ electrons in the carbon atom are hybridized into three molecular orbitals in a plane with 3-fold rotational symmetry. In the latter, the $s$ and all three $p$ valence electrons are hybridized into molecular orbitals with tetrahedral symmetry. $s p^{3}$ bonding is, e.g., found in methane and in diamond, whereas $s p^{2}$ is found in benzene and in graphite. In the $s p^{2}$ hybridization, the remaining $p$ valence electron of each carbon atom becomes part of a delocalized $\pi$-orbital, in which the electrons can move quite freely. These $\pi$-electrons have closely spaced energy levels, which allows them to absorb electromagnetic radiation over a broad spectrum. Variation of the size of the $s p^{2}$ "islands" within an $s p^{3}$ "matrix" gives rise to a characteristic variation of $n$ and $\kappa$.

The void fraction model, on the other hand, is based on the assumption that there exists strongly absorbing carbon with a single refractive index. The variation of the refractive index of LAC is explained in terms of a variable void fraction in the material. This model gives rise to a characteristic interdependence of $n$ and $\kappa$ that is distinctly different from that of the graphitisation model.

By performing a critical review of available measurements, and by comparing observations with model results, Bond and Bergstrom (2006) were able to show that a majority of observed LAC refractive indices lie along the void fraction curve. Thus they were able to constrain the range of LAC refractive indices to a rather narrow range. Most notably, they find that the refractive index of $m=1.74+0.44 i$ at a wavelength $\lambda=550 \mathrm{~nm}$, which is commonly employed in climate models, clearly lies outside the range of reasonable $m$ values of LAC. Present estimates of the climate forcing effect of LAC aerosols may therefore be in error. In the present study, we employ the refractive index values reported by Chang and Charalampopoulos (1990), which lie within the range specified by Bond and Bergstrom (2006). We consider two wavelengths, namely, $\lambda=440 \mathrm{~nm}$
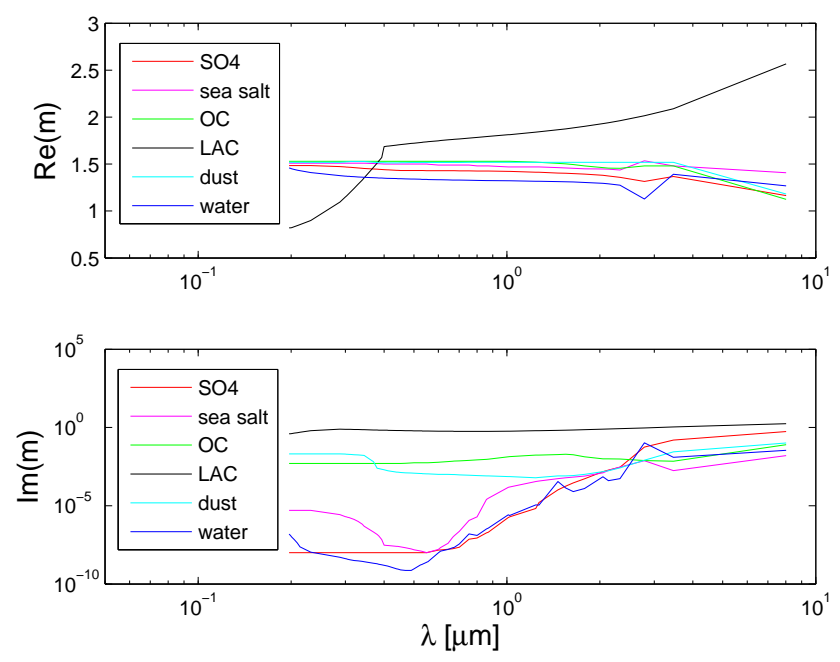

Fig. 4. Real part (top) and imaginary part (bottom) of the refractive index of sulphate (red), sea salt (purple), organic carbon (green), LAC (black), dust (cyan), and water (blue) as a function of wavelength.

and $\lambda=870 \mathrm{~nm}$, at which the corresponding refractive indices are $m=1.70+0.64 i$ and $m=1.79+0.57 i$, respectively.

For most of the other aerosol components the refractive indices are taken from the OPAC database (Hess et al., 1998), where the OPAC refractive index for soluble particles has been used for ammonium and nitrate. For organic carbon and mineral dust we have adopted the values employed in the ECHAM model. The refractive index of water is taken from Segelstein (1981). Figure 4 shows the spectral variation of the refractive indices of different aerosol components in our AOP/CTM code.

\subsection{Modelling the optical properties}

AOP computations are usually performed by computing optical properties of single particles with given chemical composition and physical properties, followed by performing an ensemble average over sizes, compositions, morphologies, and orientations. Figure 5 shows, as an example, the extinction efficiency $Q_{\text {ext }}$ of homogeneous spheres as a function of size parameter $x=k r$ for a refractive index of $m=1.6+0.001 i$ (blue line), which is typical for mineral aerosols at visible wavelengths, and corresponding results for $m=1.7+0.64 i$ (red line), which is typical for LAC at $\lambda=440 \mathrm{~nm}$. This figure clearly illustrates the quenching of internal resonances in highly absorbing material, which allows us to significantly reduce the number of discrete sizes over which we perform the size-averaging. Thus, despite the morphological complexity of LAC aggregates, the computational burden related to the size averaging is substantially reduced.

The strategy is to compute AOP for a selected number of sizes, followed by a suitable interpolation. Size-integration of AOP in conjunction with the size distribution obtained 


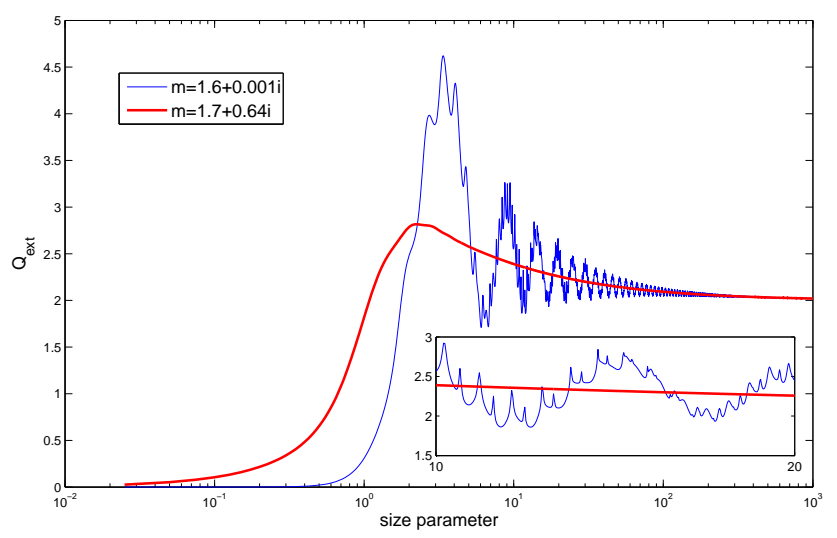

Fig. 5. Extinction efficiency $Q_{\text {ext }}$ vs. size parameter for homogeneous spheres with $m=1.6+0.001 i$ (blue line), and $m=1.7+0.64 i$ (red line).

from a CTM can then be achieved by integrating the interpolation functions weighted by the size distribution.

\section{Methods}

Optical properties of LAC are computed for $N_{\mathrm{s}}=20,40$, $60,80,100,120,140,180,220,260,300,350,650$, and 1000 , assuming a radius $a=25 \mathrm{~nm}$ of the primary monomers, and assuming a fractal structure characterized by $D_{\mathrm{f}}=1.82$ and $k_{0}=1.27$. Calculations are performed at wavelengths $\lambda=440 \mathrm{~nm}$ and $870 \mathrm{~nm}$. We used the code by $\mathrm{Xu}$ and Gustafson (2001), which computes a numerically exact solution to Maxwell's equations.

Several versions of this code exist. One version is based on computing the full $T$-matrix of the aggregates. A major advantage of this approach is that the integration of the optical properties over orientational angles can be done analytically. However, preliminary tests indicated that this version of the code does not allow us to cover the entire size range needed for this study. We therefore employed a simpler version of the code that computes the optical properties for discrete orientational angles and performs the angular integration numerically. Although less elegant and slower, this method is able to cover the required size range. It turned out that 2000 discrete angles were sufficient to obtain accurate orientationally-averaged results. Note that for larger particles or weakly absorbing particles, for which the angular distribution of the scattered radiation may display numerous sharp resonance features, the number of discrete angles required for numerical orientational averaging may be considerably larger.

For comparison, AOP computations for volume-equivalent homogeneous spheres were performed with the Mie-code written by Michael Mishchenko (http://www.giss.nasa.gov/ staff/mmishchenko).
The AOP computations were coupled to size-resolved aerosol mass mixing ratios computed with the 3-D Multiplescale Atmospheric Transport and CHemistry (MATCH) modelling system (Robertson et al., 1999; Foltescu et al., 2005; Andersson et al., 2007). We employed a version of the model which computes aerosol mass mixing ratios in four different size classes. The size classes cover particle diameters in the ranges $[0.02,0.1] \mu \mathrm{m},[0.1,1.0] \mu \mathrm{m},[1.0,2.5] \mu \mathrm{m}$, and $[2.5,10.0] \mu \mathrm{m}$. The model contains a photochemistry module which, apart from reactive gases, computes sulphate, nitrate, and ammonium concentration fields. It further contains a seasalt module and a primary particle module for computing transport and deposition of LAC, primary organic carbon $(\mathrm{OC})$, and dust. Water is treated as a diagnostic variable. The model is run with a horizontal resolution of $0.4^{\circ}$, using meteorological input data from the HIgh Resolution Limited Area Model (HIRLAM).

In the calculation of size-averaged optical properties, LAC is assumed to extend into the second size class only up to a size of $R_{\mathrm{V}}=170 \mathrm{~nm}$. Truncating the size range of LAC at a reasonable cut-off size is important in a bin representation of the size distribution in order not to over-estimate the optical cross sections. In a modal representation such a truncation is usually not necessary.

The contribution to AOP from aerosol components other than LAC is modelled by assuming that sulphate, nitrate, ammonium, seasalt, water, and the soluble fractions of OC and dust are internally mixed, homogeneous spheres. The effective refractive index of those is computed by iteratively applying the Bruggemann rule (Bruggemann, 1935) to sulphate, nitrate, ammonium, seasalt, OC, and water, and the Maxwell-Garnett rule (Maxwell-Garnett, 1904) to dust. The insoluble fraction of $\mathrm{OC}$ and dust is represented by externally mixed, homogeneous spheres. In the standard version of the code, the hydrophillic fraction of LAC is mixed by applying the Maxwell-Garnett rule. However, for the purpose of the present study, all LAC is treated as externally mixed. AOP computations for homogeneous spheres are done off-line in MATCH. In a first step, Mie computations are performed for 40000 equally-spaced size parameters $x=0.025,0.050, \ldots, 1000$, for 101 equally-spaced real parts of the refractive index $n=0.82,0.84, \ldots, 2.82$, and for 17 imaginary parts $\kappa$ of the refractive index with $\log \kappa=-9.0,-5.0,-4.0,-3.0,-2.0,-1.8, \ldots,+0.4$. In a second step, pre-computed size-averaged AOP are computed for each of the four size classes used in MATCH, and for each value of $n$ and $\kappa$. The range of refractive indices is deliberately chosen sufficiently broad to also include LAC refractive indices within a wavelength range from $\lambda=0.2 \mu \mathrm{m}$ up to $\lambda=12 \mu \mathrm{m}$ (compare with Fig. 4).

Several of the assumptions in our AOP model can introduce errors. Application of the HSA to dust is at least equally problematic as its application to LAC (Kahnert et al., 2005, 2007; Nousiainen et al., 2006; Kahnert and Nousiainen, 2006; Veihelmann et al., 2006; Kahnert and Kylling, 

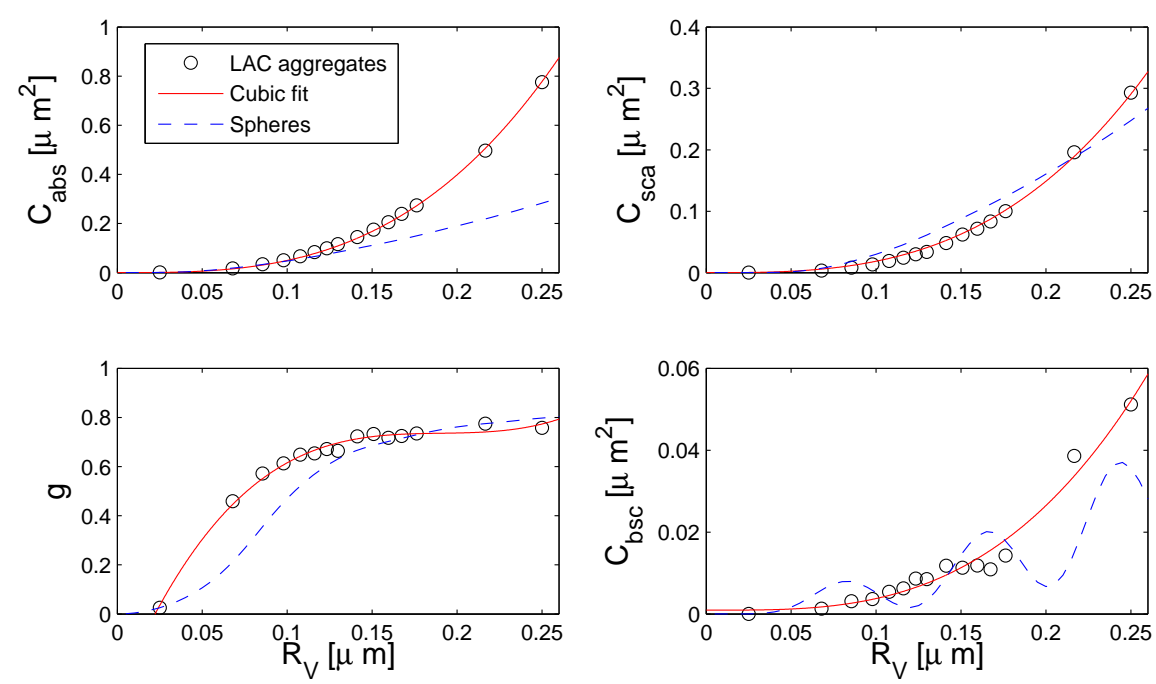

Fig. 6. Absorption cross section $C_{\text {abs }}$ (upper left), scattering cross section $C_{\text {sca }}$ (upper right), asymmetry parameter $g$ (lower left), and backscattering cross section $C_{\mathrm{bsc}}$ (lower right) as a function of volume-equivalent radius $R_{\mathrm{V}}$ at $\lambda=440 \mathrm{~nm}$ for aggregates (symbols) and homogeneous spheres (dashed blue line). The red line represents a cubic fit of the aggregate results.

2004; Kahnert, 2004; Schulz et al., 1999, 1998). Neglecting internal mixing of aged LAC is likely to underestimate its radiative forcing effect (Bond and Bergstrom, 2006). Thus, performing a detailed morphological treatment of freshly emitted LAC is only one issue in a critical assessment of the AOP model currently employed in MATCH.

The radiative aerosol properties were computed by using AOP computed in MATCH as input parameters to libRadtran (http://www.libRadtran.org), using the DIScrete Ordinate Radiative Transfer (DISORT) model (Stamnes et al., 1988) as a radiative transfer (RT) solver. RT computations were performed for (i) LAC optics treated by the aggregate model, (ii) LAC optics computed with the HSA, (iii) LAC optics accounted for by converting the recommended value of $\mathrm{MAC}=7.5 \mathrm{~m}^{2} / \mathrm{g}$ at $550 \mathrm{~nm}$ (Bond and Bergstrom, 2006) to the two wavelengths considered in this study (assuming an inverse wavelength dependency of MAC), (iv) setting the LAC mass mixing ratio equal to zero. By subtracting the radiative net flux results obtained in (iv) from each of the results obtained in (i)-(iii), one obtains the LAC radiative forcing for the three LAC optics models.

\section{Results}

\subsection{Optical properties of LAC}

Figure 6 presents optical properties of LAC computed with the aggregate model (symbols) and the HSA (dashed blue line). The absorption cross section $C_{\text {abs }}$ at $\lambda=440 \mathrm{~nm}$ (upper left) is only slightly overestimated by the HSA up to sizes of $R_{\mathrm{V}}=0.08 \mu \mathrm{m}$. At larger sizes, the HSA strongly underestimates $C_{\text {abs. }}$. The scattering cross section $C_{\text {sca }}$ (upper right) of the aggregates is overestimated by the HSA for sizes between $R_{\mathrm{V}}=0.06$ and $0.22 \mu \mathrm{m}$. The asymmetry parameter $g$ (lower left) is strongly underestimated by the HSA over much of the size range up to $R_{\mathrm{V}}=0.18 \mu \mathrm{m}$. Thus, over a large portion of the size range the homogeneous sphere model predicts a stronger influence of scattering and more side- and backscattering than the aggregate model. The lower right panel shows the backscattering cross section $C_{\mathrm{bsc}}=C_{\mathrm{sca}} p\left(180^{\circ}\right)$, where $p\left(180^{\circ}\right)$ denotes the phase function in the backscattering direction. Whereas spherical model particles display an oscillating behaviour of the backscattering cross section with particle size, the aggregate model predicts a monotonous increase. Note, however, the small amplitude fluctuations observed for the aggregate model at larger sizes. The reason for these is most likely related to the fact that the fractal parameters $D_{\mathrm{f}}$ and $k_{0}$ only define a class of aggregates. They do not unambiguously define the exact geometry of the aggregate, so for a given selection of $a, N_{\mathrm{s}}, R_{\mathrm{g}}, D_{\mathrm{f}}$, and $k_{0}$ there exists, in principle, infinitely many geometries. Whereas the cross sections $C_{\text {abs }}$ and $C_{\text {sca }}$ are rather insensitive to a variation of the geometry for fixed fractal parameters, the angular distribution of the scattered radiation, thus the backscattering cross section, is more sensitive to such a variation. One could, for the price of considerably more computation time, smooth out the fluctuation of the backscattering cross section by averaging over several geometries.

Incidentally, we verified that the optical cross sections are sufficiently constrained by specifying the values of $a, N_{\mathrm{s}}$, $R_{\mathrm{g}}, D_{\mathrm{f}}$, and $k_{0}$. For each of the aggregates up to $N_{\mathrm{s}}=100$ monomers, computations were repeated for five different geometries having the same values of the fractal parameters. The results for the optical cross sections varied by less than $1 \%$. 
Table 1. Cubic fitting coefficients and rmse for LAC optical properties at $\lambda=440 \mathrm{~nm}$.

\begin{tabular}{llllrl}
\hline & $c_{0}$ & $c_{1}$ & $c_{2}$ & $c_{3}$ & rmse \\
\hline$C_{\text {ext }}$ & - & - & - & 68.38 & $1 \times 10^{-3}$ \\
$C_{\text {sca }}$ & - & - & - & 18.61 & $4 \times 10^{-3}$ \\
$C_{\text {abs }}$ & - & - & - & 49.77 & $5 \times 10^{-3}$ \\
$g$ & -0.33 & 16.54 & -86.32 & 151.10 & $2 \times 10^{-2}$ \\
$g C_{\text {sca }}$ & 0 & -0.27 & 1.69 & 11.56 & $3 \times 10^{-3}$ \\
$C_{\text {bsc }}$ & 0 & 0 & -0.08 & 3.57 & $3 \times 10^{-3}$ \\
\hline
\end{tabular}

The LAC optical properties obtained with the aggregate model can be fitted with a Taylor series. It turns out that it is sufficient to carry the polynomial up to third order, i.e.

$\mathrm{AOP}=c_{0}+c_{1} R_{\mathrm{V}}+c_{2} R_{\mathrm{V}}^{2}+c_{3} R_{\mathrm{V}}^{3}$.

We found that $C_{\text {ext }}, C_{\text {sca }}$, and $C_{\text {abs }}$ can be fitted by setting $c_{0}=c_{1}=c_{2}=0$. Table 1 shows the resulting coefficients and the root mean square errors (rmse) of the cubic fits, obtained by least-squares error minimization. All coefficients are defined such that the resulting cross sections have units $\mu \mathrm{m}^{2}$. Note that the size-average $\langle g\rangle$ of the asymmetry parameter $g(r)$ over a size distribution $n(r)$ is defined as

$$
\langle g\rangle=\frac{1}{N\left\langle C_{\text {sca }}\right\rangle} \int n(r) C_{\text {sca }}(r) g(r) \mathrm{d} r
$$

where

$$
\begin{aligned}
\left\langle C_{\text {sca }}\right\rangle & =\frac{1}{N} \int n(r) C_{\text {sca }}(r) \mathrm{d} r \\
N & =\int n(r) \mathrm{d} r .
\end{aligned}
$$

For this reason, Table 1 provides the fitting parameters for $g C_{\text {sca }}$, which, for the purpose of computing $\langle g\rangle$, are more useful than the fitting parameters for $g$.

Note that for small homogeneous spheres the optical cross sections behave asymptotically as

$$
\begin{gathered}
C_{\mathrm{abs}} \underset{|m| R_{\mathrm{V}} \ll \lambda}{\stackrel{R_{\mathrm{V}} \ll \lambda}{\rightleftarrows}} \frac{8 \pi^{2}}{\lambda} \operatorname{Im}\left(\frac{m^{2}-1}{m^{2}+2}\right) R_{\mathrm{V}}^{3}, \\
C_{\text {sca }} \underset{|m| R_{\mathrm{V}} \ll \lambda}{\stackrel{R_{\mathrm{V}} \ll \lambda}{\rightleftarrows}} \frac{128 \pi^{5}}{3 \lambda^{4}}\left|\frac{m^{2}-1}{m^{2}+2}\right|^{2} R_{\mathrm{V}}^{6}
\end{gathered}
$$

(van de Hulst, 1981). For $C_{\text {abs }}$, this yields an asymptotic value of $c_{3}^{\text {abs }}=47.22 \mu \mathrm{m}^{-1}$ for small spheres, which is somewhat smaller than the result obtained for the aggregate model. However, the absorption cross section of homogeneous spheres in Fig. 6 cannot accurately be fitted over the entire size range by the simple ansatz $C_{\mathrm{abs}}=c_{3}^{\mathrm{abs}} R_{\mathrm{V}}^{3}$. The absorption cross sections of the larger homogeneous spheres are considerably lower than those predicted by Eq. (7). The physical reason for this is that the electromagnetic field is unable to penetrate into the centre of larger, highly absorbing spheres. This means that only the mass near the surface of the larger particles contributes to absorption. Hence, the increase of $C_{\mathrm{abs}}$ with $R_{\mathrm{V}}$ is weaker than the third power. By contrast, the geometry of fluffy aggregates is such that most of the mass interacts with the electromagnetic field, which is why the relation $C_{\mathrm{abs}} \propto R_{\mathrm{V}}^{3}$ holds over the entire size range considered here. This agrees with results by Liu and Mishchenko (2005), who computed optical properties of aggregates up to $N_{s}=400$ and observed that $C_{\text {abs }} / M$ is independent of $N_{s}$, where $M$ denotes the mass of the aggregate.

The asymptotic form for the scattering cross section given in Eq. (8) is known as the Rayleigh approximation, which is also referred to as the dipole approximation. It is based on the assumption that all electrons in the particle oscillate in phase in response to the external electromagnetic forcing, just like a dipole. This only holds for particles considerably smaller than the wavelength. In general it cannot be assumed that all monomers in our aggregate oscillate in phase, as they would in Rayleigh scattering. For this reason, Eq. (8) is not valid over the size range considered here. Clearly, the polynomial fitting ansatz is physically motivated only for $C_{\mathrm{abs}}$. For the other parameters, it is a purely mathematical approximation. The merit of this fitting approach is that the coupling of AOP and CTM computations is greatly facilitated. For instance, the output of an aerosol dynamic model with a log-normal or sectional size distribution can be easily employed in conjunction with Eq. (3) for computing size-averaged AOP, as the moments of such size distributions can be computed analytically.

From $C_{\mathrm{abs}}=c_{3}^{\text {abs }} R_{\mathrm{V}}^{3}$, we can compute the mass absorption cross section $\mathrm{MAC}=C_{\mathrm{abs}} /(\rho V)=3 c_{3}^{\mathrm{abs}} /(4 \pi \rho)$, where $V$ denotes the particle volume and $\rho$ denotes the mass density of LAC. Assuming $\rho=1.8 \mathrm{~g} \mathrm{~cm}^{-3}$, this yields $\mathrm{MAC}=6.6 \mathrm{~m}^{2} \mathrm{~g}^{-1}$. Bond and Bergstrom (2006) recommended a value of $\mathrm{MAC}=(7.5 \pm 1.2) \mathrm{m}^{2} / \mathrm{g}$ at a wavelength of $\lambda=550 \mathrm{~nm}$, and they assumed an inverse wavelength dependence of MAC (compare with Eq. 7). This yields $\mathrm{MAC}=(9.4 \pm 1.5) \mathrm{m}^{2} / \mathrm{g}$ at a wavelength of $\lambda=440 \mathrm{~nm}$. Thus, our detailed electromagnetic scattering computations for the aggregate model yield MAC results that lie below the range of MAC values recommended by Bond and Bergstrom (2006).

Figure 7 shows results analogous to those in Fig. 6, but for a wavelength of $\lambda=870 \mathrm{~nm}$. Discrepancies between the aggregate model and the HSA are even more pronounced at $\lambda=870 \mathrm{~nm}$ than at $\lambda=440 \mathrm{~nm}$. In Fig. 6 (upper left) we observed a relatively small size region in which the HSA overestimates $C_{\text {abs }}$ as compared to the aggregates. By contrast, in Fig. 7 (upper left) this effect now extends up to sizes of about $R_{V}=0.2 \mu \mathrm{m}$. Qualitatively, the graph in the upper left panel in Fig. 7 appears to be shifted to larger sizes as compared to the upper left panel in Fig. 6, which can be understood in 

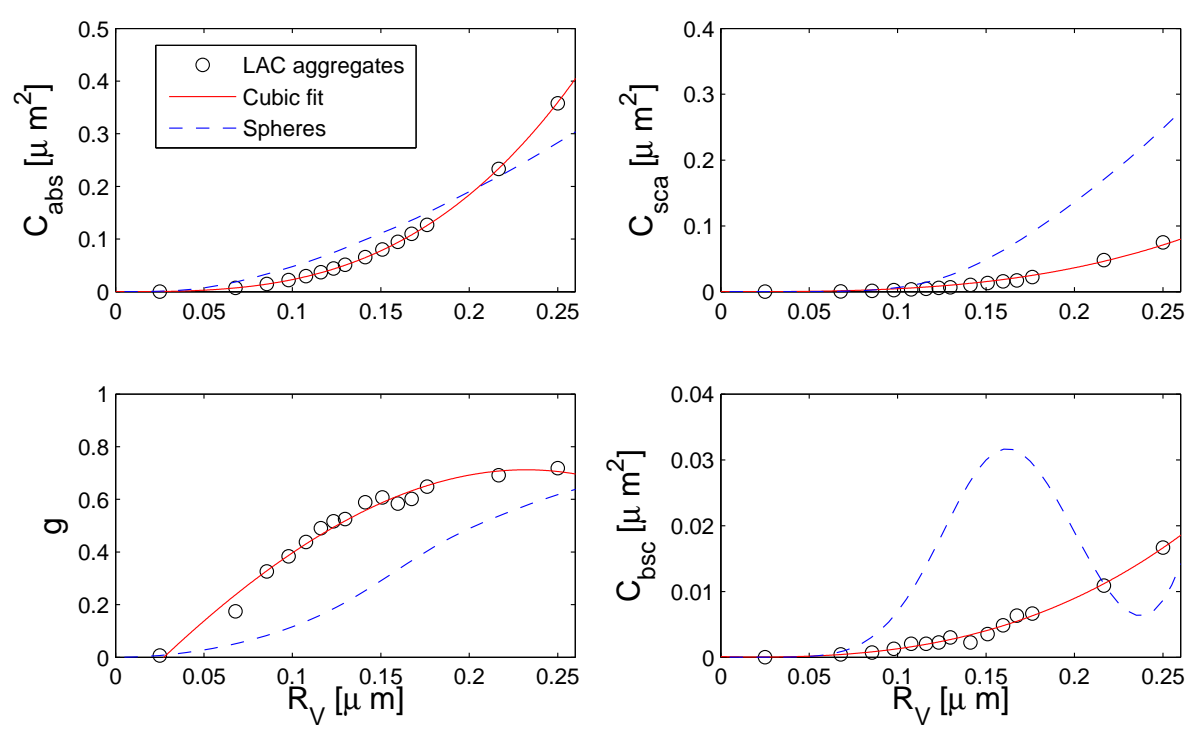

Fig. 7. As Fig. 6, but for $\lambda=870 \mathrm{~nm}$.

terms of the change in size parameters. Similar observations can be made for the lower left and right panels in Figs. 6 and 7.

The HSA results are particularly poor for those quantities that describe the scattering properties, i.e. $C_{\text {sca }}, g$, and $C_{\mathrm{bsc}}$. The poor representation of $g$ becomes clear when inspecting the phase function computed with the aggregate model and the HSA, respectively. Figure 8 gives an example for aggregates (red) and spheres (blue) with a size $R_{\mathrm{V}}=167 \mathrm{~nm}$ at $\lambda=870 \mathrm{~nm}$. Clearly, the HSA predicts less forward scattering and more side scattering, which explains the underestimation of the asymmetry parameter.

Comparing $C_{\text {abs }}$ and $C_{\text {sca }}$ in Figs. 6 and 7, we see that the single scattering albedo $\omega=C_{\mathrm{sca}} / C_{\mathrm{ext}}$ is around 0.27 at $\lambda=440 \mathrm{~nm}$, and around 0.16 at $\lambda=870 \mathrm{~nm}$. Bond and Bergstrom (2006) recommended to assume a value of $\omega=0.25$ at $\lambda=550 \mathrm{~nm}$.

Table 2 presents the cubic fitting parameters of the optical properties computed with the aggregate model at $\lambda=870 \mathrm{~nm}$. Here we obtain $c_{3}^{\text {abs }}=23.01 \mu \mathrm{m}^{-1}$, from which we derive $\mathrm{MAC}=3.1 \mathrm{~m}^{2} / \mathrm{g}$. Assuming an inverse wavelength dependence of MAC, the recommended value of Bond and Bergstrom (2006) corresponds to $\mathrm{MAC}=(4.7 \pm 0.8) \mathrm{m}^{2} / \mathrm{g}$ at a wavelength of $\lambda=870 \mathrm{~nm}$. Again, our result obtained with the aggregate model lies below this range.

We noted earlier that the electromagnetic field inside a highly absorbing large sphere is so quickly attenuated that it does not penetrate to the sphere's core. As a consequence, the mass near the centre of the sphere is not able to contribute much to the total absorption. One would therefore expect that a hollow sphere with an LAC shell of outer radius $R_{\text {shell }}$ and an empty inner core of radius $R_{\text {core }}=\left(R_{\text {shell }}^{3}-R_{\mathrm{V}}^{3}\right)^{1 / 3}$ should have a higher absorption cross section than a mass-equivalent

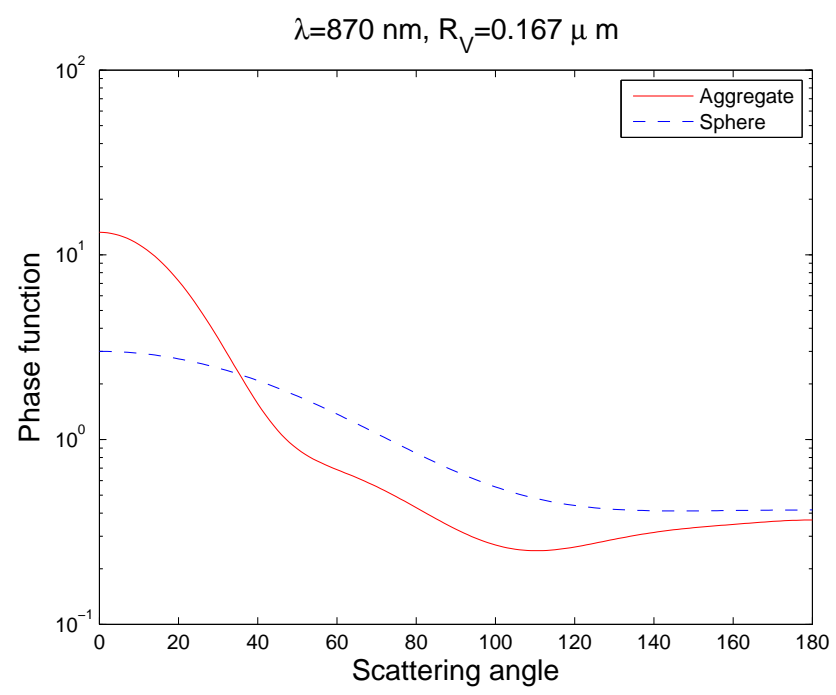

Fig. 8. Phase function of aggregates (red solid line) and homogeneous spheres (blue dashed line) with a size $R_{\mathrm{V}}=167 \mathrm{~nm}$ at a wavelength of $\lambda=870 \mathrm{~nm}$.

homogeneous sphere of radius $R_{\mathrm{V}}$. As this hollow-sphere model distributes more mass near the surface, the electromagnetic field can interact with and be absorbed by a larger amount of the particle's mass. Also, the hollow sphere has a larger physical cross section than the homogeneous sphere of equal mass. We test this idea by computing the optical properties of hollow spheres with the electromagnetic scattering code written by Toon and Ackermann (1981). Results are shown in Fig. 9. The solid line shows $C_{\text {abs }}$ (upper left) and $C_{\text {sca }}$ (lower left) of a hollow sphere as a function of the outer shell radius $R_{\text {shell }}$ at a wavelength of $440 \mathrm{~nm}$ and for 
Table 2. As Table 1 , but for $\lambda=870 \mathrm{~nm}$.

\begin{tabular}{llllrl}
\hline & $c_{0}$ & $c_{1}$ & \multicolumn{1}{l}{$c_{2}$} & $c_{3}$ & \multicolumn{1}{l}{ rmse } \\
\hline$C_{\text {ext }}$ & - & - & - & 27.55 & $2 \times 10^{-3}$ \\
$C_{\text {sca }}$ & - & - & - & 4.54 & $3 \times 10^{-3}$ \\
$C_{\text {abs }}$ & - & - & - & 23.01 & $1 \times 10^{-3}$ \\
$g$ & -0.18 & 6.88 & -9.56 & -15.09 & $3 \times 10^{-2}$ \\
$g C_{\text {sca }}$ & 0 & 0.02 & -0.70 & 5.97 & $6 \times 10^{-4}$ \\
$C_{\text {bsc }}$ & 0 & 0.01 & 0.10 & 0.75 & $5 \times 10^{-4}$ \\
\hline
\end{tabular}
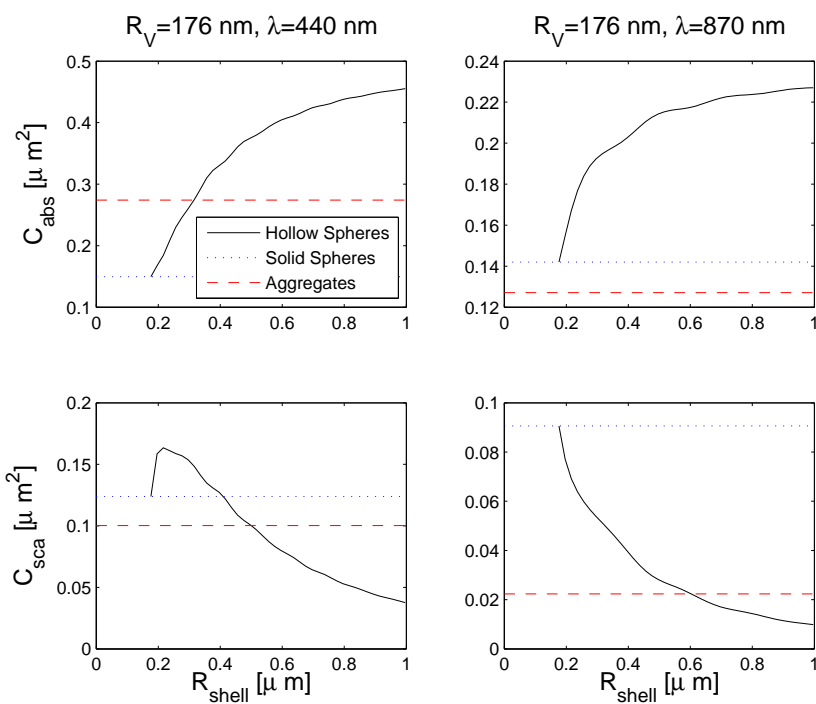

Fig. 9. Absorption cross section $C_{\text {abs }}$ (upper row) and scattering cross section $C_{\text {sca }}$ (lower row) for a hollow LAC sphere as a function of the outer shell radius $R_{\text {shell }}$ (solid lines). The particles have a constant mass corresponding to a volume-equivalent radius of $R_{\mathrm{V}}=176 \mathrm{~nm}$. Results are presented for wavelengths of $440 \mathrm{~nm}$ (left column) and $870 \mathrm{~nm}$ (right column). For comparison, corresponding results for massive spheres $\left(R_{\text {shell }}=R_{\mathrm{V}}\right)$ and aggregates are indicated by dotted and dashed lines, respectively.

$R_{\mathrm{V}}=176 \mathrm{~nm}$. For comparison, the dotted and dashed lines show the corresponding results for the homogeneous sphere and the aggregate at this size, respectively. One observes indeed that by increasing $R_{\text {shell }}$ one can increase $C_{\text {abs }}$. At $R_{\text {shell }}=310 \mathrm{~nm}$, the absorption cross sections of the hollow sphere and the aggregate coincide.

However, there are two major problems with the hollowsphere model. First, this model overestimates the scattering cross section at $R_{\text {shell }}=310 \mathrm{~nm}$ by about $50 \%$. So it fails to predict a correct single scattering albedo. Second, the hollow sphere model does not consistently produce reasonable results for $C_{\mathrm{abs}}$ at other wavelengths. The upper and lower right panels in Fig. 9 show $C_{\mathrm{abs}}$ and $C_{\mathrm{sca}}$, respectively, for a wavelength of $870 \mathrm{~nm}$. At this wavelength, both the solid and hollow sphere models overestimate the absorption cross sec-

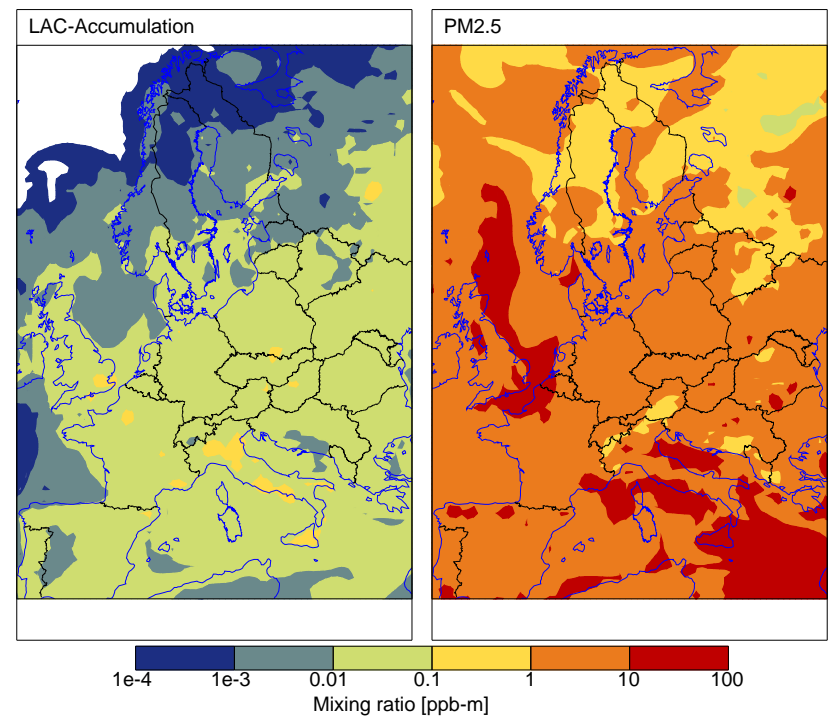

Fig. 10. MATCH results for $\mathrm{LAC}$ (left) and $\mathrm{PM}_{2.5}$ (right) mass mixing ratios in the lowest model layer on 25 July 2006.

tion of the aggregate for all values of $R_{\text {shell }}$. The comparison of the aggregate results with those obtained for the hollow spheres underlines the fundamental difficulties encountered when trying to model the AOP of aggregated particles with simple geometries.

\subsection{Radiative properties of LAC}

One advantage of the cubic fitting approach given in Eq. (3) is that for sectional or log-normal aerosol size distributions models the integration of AOP over size can be done analytically. Thus, coupling the AOP results obtained with the aggregate model to the output of a CTM becomes a fairly simple task.

Figure 10 shows an example of mass mixing ratios of LAC in the second size class (left) and $\mathrm{PM}_{2.5}$ (right) computed with MATCH for 25 July 2006. $\mathrm{PM}_{2.5}$ denotes the total mass of aerosols with diameters up to $2.5 \mu \mathrm{m}$. The figure shows results for the lowest model layer (between ca. 0-60 m). Although LAC only contributes a few percent to the total aerosol mass, it can contribute significantly to the aerosol optical depth owing to its high extinction cross section.

We pick a location south of Paris at a latitude of $48.2^{\circ} \mathrm{N}$ and a longitude of $2.3^{\circ} \mathrm{E}$. So we choose a location that lies near, but not directly within one of the major source regions of LAC emissions in Europe. Figure 11 shows vertical profiles of the extinction efficiency computed by coupling the MATCH aerosol profile at this location (see Fig. 10) to the results from the AOP computations. The upper and lower panels correspond to wavelengths of $\lambda=440 \mathrm{~nm}$ and $\lambda=870 \mathrm{~nm}$, respectively. The extinction efficiency is defined as $\mathrm{d} \tau / \mathrm{d} z$, where $\tau(z)=\int_{z_{0}}^{z}\left\langle C_{\text {ext }}\right\rangle(\zeta) N(\zeta) \mathrm{d} \zeta$ is the extinction optical 

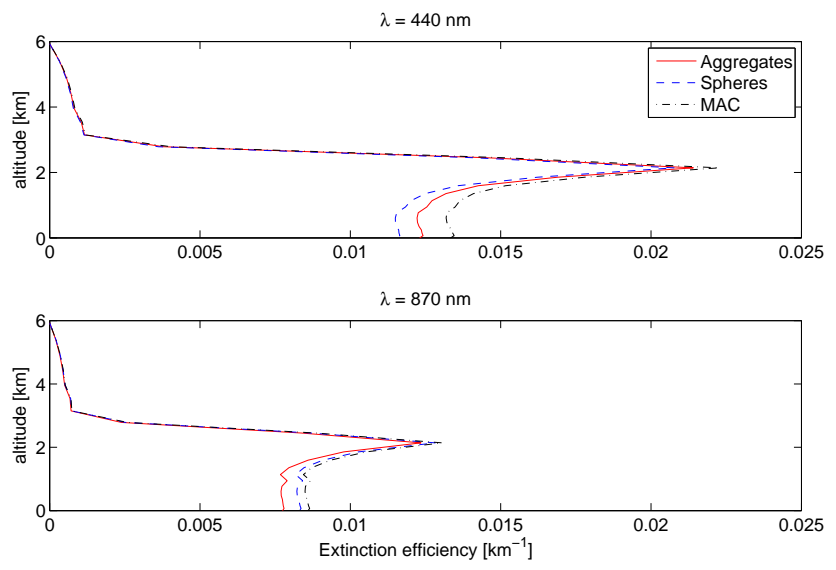

Fig. 11. Total aerosol extinction efficiency as a function of altitude at $\lambda=440 \mathrm{~nm}$ (upper panel) and $\lambda=870 \mathrm{~nm}$ (lower panel). The contribution of LAC has been computed by the aggregate model (red solid line), the HSA (dashed blue line), and assuming a constant MAC (dash-dotted black line).

depth at altitude $z,\left\langle C_{\text {ext }}\right\rangle$ denotes the ensemble-averaged extinction cross section, and $N$ is the aerosol number density. Note that $\mathrm{d} \tau / \mathrm{d} z$ in Fig. 11 contains contributions from all chemical aerosol components in all four size classes (up to diameters of $10 \mu \mathrm{m})$. The contribution of LAC has been computed with the aggregate model (red solid line), the HSA (dashed blue line), and assuming a constant MAC (dashdotted black line).

As expected, the constant-MAC approach yields the highest extinction efficiencies. At $\lambda=440 \mathrm{~nm}$, the HSA yields lower values for $\mathrm{d} \tau / \mathrm{d} z$ than the aggregate model. At $\lambda=870 \mathrm{~nm}$, the order is reversed. This is what we expect from our previous discussion of $C_{\mathrm{abs}}$ and $C_{\mathrm{sca}}$ in Figs. 6 and 7. Note also the pronounced maximum at $2 \mathrm{~km}$, which is caused by the presence of an aerosol layer at this altitude.

The vertical profiles of the extinction efficiency, the single scattering albedo, and the asymmetry parameter are taken as input parameters to libRadtran, with which we compute the spectral radiative net flux $F_{\lambda}=F_{\lambda}^{\mathrm{s}}+F_{\lambda}^{-}-F_{\lambda}^{+}$. Here $F_{\lambda}^{\mathrm{s}}$ denotes the direct solar flux, i.e. that part of the incoming flux that survives extinction, $F_{\lambda}^{-}$represents the diffuse downwelling flux, and $F_{\lambda}^{+}$is the upwelling flux. We assume a solar zenith angle of $35^{\circ}$. Repeating these computations in the absence of LAC, we obtain a spectral net flux $F_{\lambda}^{0}$ for an LAC-free atmosphere, from which we can compute the spectral radiative forcing effect $\Delta F_{\lambda}$ of LAC according to

$\Delta F_{\lambda}=F_{\lambda}-F_{\lambda}^{0}$.

Figure 12 shows the resulting profiles of $\Delta F_{\lambda}$ at $\lambda=440 \mathrm{~nm}$ (upper panel) and $\lambda=870 \mathrm{~nm}$ (lower panel). The high absorption due to LAC causes a pronounced positive radiative forcing effect in and above the aerosol layer. As a consequence, less solar radiation can penetrate down to the ground, resulting in a negative forcing effect below the
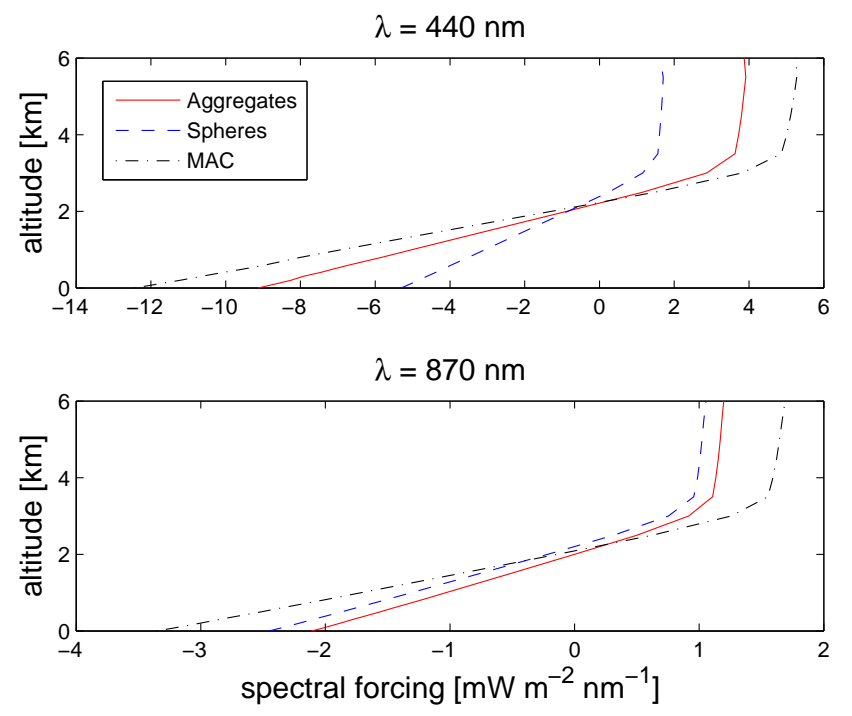

Fig. 12. As Fig. 11, but for the spectral radiative forcing effect of LAC, assuming a solar zenith angle of $35^{\circ}$.

aerosol layer. Table 3 shows spectral forcing at top of atmosphere (TOA) obtained with the three LAC-optics models.

At $\lambda=440 \mathrm{~nm}$, the HSA under-predicts spectral forcing at TOA by roughly a factor $1 / 2$ as compared to the aggregate model, whereas the constant-MAC prediction lies $37 \%$ above the result computed with the aggregate model. In the preceding discussion of Fig. 6, it was noted that the HSA overpredicts the effect of scattering. In fact, at $\lambda=440 \mathrm{~nm}$ the HSA yields single-scattering albedos of $\omega=0.46$, whereas the aggregate model predicts only $\omega=0.27$. In addition, the HSA overestimates the amount of side- and backscattering. However, scattering counteracts the positive forcing effect owing to absorption. Thus, the high HSA-error in TOA spectral forcing is the combined effect of too little absorption and too much scattering.

The situation is seemingly different at $\lambda=870 \mathrm{~nm}$. The HSA results shown in the lower panel of Fig. 12 lie rather close to those obtained with the aggregate model. However, the origin of this agreement is not a better performance of the HSA, but a rather lucky error cancellation. As can be seen in Fig. 7, the agreement between the AOP modelled with the HSA and with the aggregate model is considerably worse than that between the corresponding results shown in Fig. 6. The HSA yields a single-scattering albedo of $\omega=0.39$, whereas the aggregate model predicts a value of only $\omega=0.16$. Further, the HSA considerably underestimates the asymmetry parameter $g$. Thus, the HSA overestimates the negative cooling effect due to scattering. At the same time, the HSA overestimates the positive forcing effect due to absorption. The net effect of these two large errors with opposite signs is that the spectral forcing computed with the HSA shows a coincidental agreement with that computed with the aggregate model. 
Table 3. Spectral forcing in $\mathrm{mW} \mathrm{m}^{-2} \mathrm{~nm}^{-1}$ at TOA computed with the three different LAC-optics models.

\begin{tabular}{lll}
\hline & $\lambda=440 \mathrm{~nm}$ & $\lambda=870 \mathrm{~nm}$ \\
\hline Aggregates & 3.8 & 1.2 \\
Spheres & 1.6 & 1.0 \\
MAC & $5.2 \pm 0.9$ & $1.7 \pm 0.3$ \\
\hline
\end{tabular}

It can be difficult to think in terms of spectral forcing rates. It may help to gauge the results shown in Fig. 12 by comparing them to broadband results. The solar broadband flux is $F^{\mathrm{s}}=1373 \mathrm{~W} \mathrm{~m}^{-2}$. Global broadband forcing estimates of LAC range between 0.4 to $1.2 \mathrm{~W} \mathrm{~m}^{-2}$ (Forster et al., 2007), which is $0.3-0.9 \%$ of the solar flux. The solar spectral flux at the two wavelengths we considered is $F_{440}^{\mathrm{s}}=1722 \mathrm{~mW} \mathrm{~m}^{-2} \mathrm{~nm}^{-1}$ and $F_{870}^{\mathrm{s}}=986 \mathrm{~mW} \mathrm{~m}^{-2} \mathrm{~nm}^{-1}$, respectively. Thus the spectral forcing results obtained with the aggregate model (see Table 3 ) correspond to $2 \%$ of the solar spectral flux at $\lambda=440 \mathrm{~nm}$, and to $1 \%$ at $\lambda=870 \mathrm{~nm}$. These are rather high percentages compared to the global broadband forcing estimates of LAC. However, it is important to keep in mind that our spectral forcing estimates are based on modelling a local situation near a polluted area.

\section{Conclusions}

The main message of this study is that it is feasible to treat optical properties of freshly emitted LAC within both chemical data assimilation and climate modelling by use of numerically exact solutions to Maxwell's equations. This will help to constrain the radiative forcing effect of LAC, and to construct accurate observation operators for assimilating remote sensing observations into chemical transport models. As the optical properties for sub-wavelength sized, highly absorbing aerosols are relatively smooth functions of size, it is sufficient to perform computations of optical properties at each wavelength for a relatively small number of discrete sizes, between which one can interpolate with cubic fitting polynomials. The results are easily coupled to aerosol size distributions modelled with an aerosol chemical transport model, thus obtaining 3-D fields of size-averaged optical properties. Those can subsequently be included in the observation operator of a chemical data assimilation algorithm, or, as demonstrated in this study, coupled to radiative transfer computations to simulate the direct radiative forcing effect of aerosols.

Another important message of this investigation is that the use of the homogeneous sphere approximation introduces errors in radiative forcing computations that can, depending on the wavelength range, underestimate the positive forcing effect of LAC by as much as a factor of $1 / 2$.
An important methodological observation was that the use of a $T$-matrix based analytical computation of orientationally averaged aerosol optical properties was numerically too limited to cover the relevant range of size parameters. In many applications this approach is superior to other formulations of the electromagnetic scattering problem, as it significantly expedites the computation of orientationally-averaged optical properties (Mishchenko, 1991; Khlebtsov, 1992). However, this version of the code by $\mathrm{Xu}$ and Gustafson (2001) was too limited in the range of accessible size parameters owing to its high memory requirements. A simpler version of the same code, which computes the optical properties for discrete orientational angles and performs the angular integration numerically, proved to be sufficiently fast and stable for our task. This corresponds to similar observations by Okada et al. (2008) made for silicate aggregates in conjunction with the code by Mackowski and Mishchenko (1996).

One advantage of the code by Mackowski and Mishchenko (1996) is that there exists a version by Okada and Kokhanovsky (2009) in which the numerical integration over orientations is parallelized. A potential advantage of the code by $\mathrm{Xu}$ and Gustafson (2001) is that it allows for limiting the range of interactions between distant monomers in the aggregate, which may be exploited to reduce computation time.

Optical and radiative properties computed with the aggregate model have been compared to corresponding results obtained by the homogeneous sphere approximation (HSA), and to results assuming a prescribed mass absorption cross section (MAC). Clearly, the HSA is far from being state-ofthe-art, as the Rayleigh-Debeye-Gans (RDG) approximation (Sorensen, 2001) is known to yield more accurate results for the optical properties of aggregated particles. However, comparison of the aggregate model with the HSA model is not only of historical, but above all of didactic value. Since use of the HSA is still common practice in the climate modelling community, the test cases presented in this study clearly illustrate the potentially high errors one introduces with the HSA in modelling the radiative forcing effect of LAC in the atmosphere.

The comparisons between the results obtained with the aggregate model and with the prescribed MAC-values may have to be taken with a grain of salt. The aggregate model is, to be sure, based on rigorous theory, and it uses realistic aggregate geometries. Hence, this approach eliminates errors related to approximations made in the homogeneous sphere model or in the RDG approach. Thus, results obtained with the aggregate model can be taken as an "exact" reference for relative comparisons with theoretical approximations or simplified geometrical models. However, the aggregate model still needs to rely on assumptions about the refractive index of LAC and its spectral dependence, as well as on the size of the primary monomers in the aggregate, both of which can vary depending on the combustion conditions under which the LAC aggregates are formed. Thus, the results obtained with the aggregate model cannot be interpreted as an absolute 
exact reference. To gauge the performance of the aggregate model in comparison to the range of MAC values recommended by Bond and Bergstrom (2006), more detailed studies are needed to test the sensitivity of the optical and radiative properties of the aggregates to variations in the refractive index and the primary monomer size.

We may speculate at this point what kind of outcome one can expect from such a sensitivity study. If we keep the mass per aggregate constant and decrease the monomer radius $a$, then the number of monomers $N_{\mathrm{s}}$ will increase. It has been noted by Bond and Bergstrom (2006) that MAC is completely insensitive to a variation in $a$ for monomer radii smaller than $40 \mathrm{~nm}$ (diameters smaller than $80 \mathrm{~nm}$ ). So one would not anticipate MAC to change appreciably by decreasing $a$ while increasing $N_{\mathrm{s}}$. At the same time, such a variation is likely to change the scattering cross section, hence the single scattering albedo. To obtain higher values of MAC it seems to be most promising to increase $|m|$.

It is emphasised, once more, that the fitting ansatz employed for the cross sections is physically well motivated for $C_{\text {abs }}$, but only approximately valid for $C_{\text {sca }}$. The constancy of the single scattering albedo $\omega$ resulting from this ansatz usually breaks down at very small particle sizes. In fact, from Eqs. (7) and (8), one can see that $\omega=C_{\mathrm{sca}} /\left(C_{\mathrm{sca}}+C_{\mathrm{abs}}\right)$ should, for small $R_{\mathrm{V}}$, go to zero as $\sim R_{\mathrm{V}}^{3}$. Instead of fitting $C_{\text {abs }}$ and $C_{\text {sca }}$, it may be a more accurate approach to fit $C_{\mathrm{abs}}$ and $\omega$, where one would have to find a suitable fitting function for $\omega$.

Finally, one may question the measure of size-equivalence employed in this study. The classical approach in simulating the optical properties of complex-shaped particles is to employ model geometries that are as simple as possible, and to find a suitable measure of size-equivalence such that the optical properties of the model particles agree with those of the complex reference particles as closely as possible. One may, as in this study, define size-equivalence in terms of equal mass/volume. Alternatively, one may consider particles to have equivalent size if they have the same average projected surface area. For aggregates, one may also employ the radius of gyration as a size measure. Grenfell and Warren (1999) considered the optical properties of ice particles and argued that for getting the most out of spherical model particles one should use spheres that have the same area-tovolume ratio as the nonspherical ice crystals. Other measures of size-equivalence could be contrived, and it is possible that one could find a suitable measure that improves the HSA results in comparison to the aggregate model results. One problem with this approach is that measures of size-equivalence that perform well at one wavelength tend to perform worse at others (Kahnert et al., 2007). The main problem within the context of our study is that the ansatz of manipulating the measure of size equivalence does not represent an acceptable option in chemical data assimilation. Choosing any measure of size-equivalence other than the equivalent mass/volume radius $R_{\mathrm{V}}$ implies that the model particles (e.g. spheres) have a different mass per particle than the reference particles (e.g. aggregates). Thus, in order that the total aerosol mass mixing ratio remains constant, one needs to change the aerosol number density $n(r)$ accordingly. However, in an aerosol dynamic model the aerosol mass and the aerosol number distribution are the prognostic variables of the model. The aerosol optical properties are merely diagnostic variables employed in assimilating observations. The purpose of assimilation is to improve the model prediction of the prognostic variables (Kahnert, 2008), or to inversely model the observations in the space of the prognostic model variables (Kahnert, 2009). Introducing measures of size-equivalence other than $R_{\mathrm{V}}$ would mean to discard one of the main prognostic model variables in order to make the computation of auxiliary optical variables somewhat more convenient. Thus, an aerosol optics model that is to be useful in conjunction with aerosol dynamic CTM computations should be based on employing model particles with equivalent volume radius $R_{\mathrm{V}}$. This constraint can make AOP modelling coupled to CTM computations particularly challenging.

Acknowledgements. The author thanks D. Mackowski for providing his program for aggregated-particle generation. Y.-L. Xu and B. Gustafson are acknowledged for making their electromagnetic scattering program publicly available. M. Mishchenko is acknowledged for making his Lorenz-Mie program publicly available. O. Toon and T. Ackerman are acknowledged for making their coated-sphere code available. This work was supported by the Swedish Research Council under contract number 80438701 .

Edited by: E. Weingartner

\section{References}

Andersson, C., Langner, J., and Bergström, R.: Interannual variation and trends in air pollution over Europe due to climate variability during 1958-2001 simulated with a regional CTM coupled to the ERA40 reanalysis, Tellus B, 59, 77-98, 2007.

Bond, T. C. and Bergstrom, R. W.: Light absorption by carbonaceous particles: an investigative review, Aerosol Sci. Technol., 40, 27-67, 2006.

Bond, T. C., Habib, G., and Bergstrom, R. W.: Limitations in the enhancement of visible light absorption due to mixing state, J. Geophys. Res., 111, D20211, doi:10.1029/2006JD007315, 2006.

Borghese, F., Denti, P., Saija, R., Toscano, G., and Sindoni, O. I.: Use of group theory for the description of electromagnetic scattering from molecular systems, J. Opt. Soc. Am. A, 1, 183-191, 1984.

Bruggemann, D. A. G.: Berechnung verschiedener physikalischer Konstanten von heterogenen Substanzen. 1. Dielektrizitätskonstanten und Leitfähigkeiten der Mischkörper aus isotropen Substanzen, Ann. Phys., 24, 636-664, 1935.

Chang, H. and Charalampopoulos, T. T.: Determination of the wavelength dependence of refractive indices of flame soot, Proc. R. Soc. Lond. A, 430, 577-591, 1990.

Chýlek, P., Videen, G., Geldart, D. J. W., Dobbie, J. S., and Tso, H. C. W.: Effective medium approximations for heterogeneous 
particles, in: Light scattering by nonspherical particles, edited by: Mishchenko, M. I., Hovenier, J. W., and Travis, L. D., 274308, Academic Press, San Diego, USA, 2000.

Dubovik, O., Holben, B. N., Lapyonok, T., Sinyuk, A., Mishchenko, M. I., Yang, P., and Slutsker, I.: Non-spherical aerosol retrieval method employing light scattering by spheroids, Geophys. Res. Lett., 29(10), 1415, doi:10.1029/2001GL014506, 2002.

Färnlund, J., Holman, C., and Kågeson, P.: Emissions of ultrafine particles from different types of light duty vehicles, Tech. Rep. 2001:10, Swedish National Road Administration, Borlänge, 2001.

Foltescu, V., Pryor, S. C., and Bennet, C.: Sea salt generation, dispersion and removal on the regional scale, Atmos. Environ., 39, 2123-2133, 2005.

Forster, P., Ramaswamy, V., Artaxo, P., Berntsen, T., Betts, R., Fahey, D. W., Haywood, J., Lean, J., Lowe, D. C., Myhre, G., Nganga, J., Prinn, R., Raga, G., Schulz, M., and Van Dorland, R. V.: Changes in atmospheric constituents and in radiative forcing, in: Climate Change 2007: The Physical Science Basis., edited by: Solomon, S., Qin, D., Manning, M., Chen, Z., Marquis, M., Averyt, K. B., Tignor, M., and Miller, H. L., Contribution of Working Group I to the Fourth Assessment Report of the Intergovernmental Panel on Climate Change, Cambridge University Press, Cambridge, 2007.

Fuller, K. A., Malm, W. C., and Kreidenweis, S. M.: Effects of mixing on extinction by carbonaceous particles, J. Geophys. Res., 104, 15941-15954, 1999.

Grenfell, T. C. and Warren, S. G.: Representation of a nonspherical ice particle by a collection of independent spheres for scattering and absorption of radiation, J. Geophys. Res., 104, 3169731709, 1999.

Hess, M., Koepke, P., and Schult, I.: Optical properties of aerosols and clouds: The software package OPAC, B. Am. Meteor. Soc., 79, 831-844, 1998.

Jacobson, M. Z.: A physically-based treatment of elemental carbon optics: Implications for global direct forcing of aerosols, Geophys. Res. Lett, 27, 217-220, 2000.

Jones, A. R.: Light scattering in combustion, in: Light Scattering Reviews, edited by: Kokhanovsky, A., Springer, Berlin, 2006.

Kahnert, F. M.: Reproducing the optical properties of fine desert dust aerosols using ensembles of simple model particles, J. Quant. Spectrosc. Radiat. Transfer, 85, 231-249, 2004.

Kahnert, M.: Variational data analysis of aerosol species in a regional CTM: background error covariance constraint and aerosol optical observation operators, Tellus B, 60, 753-770, 2008.

Kahnert, M.: On the observability of chemical and physical aerosol properties by optical observations: Inverse modelling with variational data assimilation, Tellus B, 61, 747-755, 2009.

Kahnert, M. and Kylling, A.: Radiance and flux simulations for mineral dust aerosols: Assessing the error due to using spherical or spheroidal model particles, J. Geophys. Res., 109, D09203, doi:10.1029/2003JD004318, errata: doi:10.1029/2004JD005311, 2004.

Kahnert, M. and Nousiainen, T.: Uncertainties in measured and modelled asymmetry parameters of mineral dust aerosols, J. Quant. Spectrosc. Radiat. Transfer, 100, 173-178, 2006.

Kahnert, M., Nousiainen, T., and Veihelmann, B.: Spherical and spheroidal model particles as an error source in aerosol climate forcing and radiance computations: a case study for feldspar aerosols, J. Geophys. Res., 110, D18S13, doi:10.1029/2004JD005558, 2005.

Kahnert, M., Nousiainen, T., and Räisänen, P.: Mie simulations as an error source in mineral aerosol radiative forcing calculations, Q. J. Roy. Meteor. Soc., 133, 299-307, 2007.

Khlebtsov, N. G.: Orientational averaging of light-scattering observables in the T-matrix approach, Appl. Opt., 31, 5359-5365, 1992.

Kocifaj, M. and Videen, G.: Optical behavior of composite carbonaceous aerosols: DDA and EMT approaches, J. Quant. Spectrosc. Radiat. Transfer, 109, 1404-1416, 2008.

Liu, L. and Mishchenko, M. I.: Effects of aggregation on scattering and radiative properties of soot aerosols, J. Geophys. Res., 110, D11211, doi:10.1029/2004JD005649, 2005.

Liu, L., Mishchenko, M. I., and Arnott, W. P.: A study of radiative properties of fractal soot aggregates using the superposition $\mathrm{T}$ matrix method, J. Quant. Spectrosc. Radiat. Transfer, 109, 26562663, 2008.

Mackowski, D. W.: Calculation of total cross sections of multiplesphere clusters, J. Opt. Soc. Am. A., 11, 2851-2861, 1994.

Mackowski, D. W.: A simplified model to predict the effects of aggregation on the absorption properties of soot aggregates, J. Quant. Spectrosc. Radiat. Transfer, 100, 237-249, 2006.

Mackowski, D. W. and Mishchenko, M. I.: Calculation of the T matrix and the scattering matrix for ensembles of spheres, J. Opt. Soc. Am. A., 13, 2266-2278, 1996.

Maxwell-Garnett, J. C.: Colours in metal glasses and in metallic films, Philos. Trans. R. Soc. A., 203, 385-420, 1904.

Mie, G.: Beiträge zur Optik trüber Medien, speziell kolloidaler Metallösungen, Ann. Phys., 25, 377-445, 1908.

Mishchenko, M. I.: Light scattering by randomly oriented axially symmetric particles, J. Opt. Soc. Am. A., 8, 871-882, 1991.

Mishchenko, M. I., Travis, L. D., and Mackowski, D. W.: T-matrix computations of light scattering by nonspherical particles: a review, J. Quant. Spectrosc. Radiat. Transfer, 55, 535-575, 1996.

Mishchenko, M. I., Travis, L. D., Kahn, R. A., and West, R. A.: Modeling phase functions for dustlike tropospheric aerosols using a shape mixture of randomly oriented polydisperse spheroids, J. Geophys. Res., 102, 16831-16847, 1997.

Nousiainen, T., Kahnert, M., and Veihelmann, B.: Light scattering modeling of small feldspar aerosol particles using polyhedral prisms and spheroids, J. Quant. Spectrosc. Radiat. Transfer, 101, 471-487, 2006.

Okada, Y. and Kokhanovsky, A. A.: Light scattering and absorption by densely packed groups of spherical particles, J. Quant. Spectrosc. Radiat. Transfer, 110, 902-917, 2009.

Okada, Y., Mukai, T., Mann, I., Nomura, H., Takeuchi, T., Sano, I., and Mukai, S.: Grouping and adding method for calculating light scattering by large fluffy aggregates, J. Quant. Spectrosc. Radiat. Transfer, 108, 65-80, 2007.

Okada, Y., Mann, I., Mukai, T., and Köhler, M.: Extended calculation of polarization and intensity of fractal aggregates based on rigorous method for light scattering simulations with numerical orientation averaging, J. Quant. Spectrosc. Radiat. Transfer, 109, 2613-2627, 2008.

Otto, S., Bierwirth, E., Weinzierl, B., Kandler, K., Esselborn, M., Tesche, M., Schladitz, A., Wendisch, M., and Trautmann, T.: Solar radiative effects of a Saharan dust plume observed during SA- 
MUM assuming spheroidal model particles, Tellus B, 61, 270296, 2009.

Pilinis, C. and Li, X.: Particle shape and internal inhomogeneity effects in the optical properties of tropospheric aerosols of relevance to climate forcing, J. Geophys. Res., 103, 3789-3800, 1998.

Robertson, L., Langner, J., and Enghardt, M.: An Eulerian limitedarea atmospheric transport model, J. Appl. Meteorol., 38, 190210, 1999.

Rother, T., Schmidt, K., Wauer, J., Shcherbakov, V., and Gaeyt, J.F.: Light scattering on Chebyshev particles of higher order, Appl. Opt., 45, 6030-6037, 2006.

Schulz, F. M., Stamnes, K., and Stamnes, J. J.: Modeling the radiative transfer properties of media containing particles of moderately and highly elongated shape, Geophys. Res. Lett., 25, 44814484, 1998.

Schulz, F. M., Stamnes, K., and Stamnes, J. J.: Shape-dependence of the optical properties in size-shape distributions of randomly oriented prolate spheroids, including highly elongated shapes, J. Geophys. Res., 104, 9413-9421, 1999.

Segelstein, D.: The Complex Refractive Index of Water, Master thesis, University of Missouri-Kansas City, 1981.

Smekens, A., Pauwels, J., Berghmans, P., and Grieken, R. V.: Correlation study between the aerodynamic diameter and the number of primary particles of soot aggregates by STEM, J. Aerosol Sci., 28, 761-762, 1997.

Sorensen, C. M.: Light scattering by fractal aggregates: a review, Aerosol Sci. Technol., 35, 648-687, 2001.

Sorensen, C. M. and Roberts, G. M.: The prefactor of fractal aggregates, J. Colloid. Interface Sci., 186, 447-452, 1997.
Stamnes, K., Tsay, S.-C., Wiscombe, W., and Jayaweera, K.: Numerically stable algorithm for discrete-ordinate-method radiative transfer in multiple scattering and emitting layered media, Appl. Opt., 27, 2502-2509, 1988.

Toon, O. B. and Ackermann, T. P.: Algorithms for the calculation of scattering by stratified spheres, Appl. Opt., 20, 3657-3660, 1981.

van de Hulst, H. C.: Light Scattering by Small Particles, Dover Publications, Inc., New York, USA, p. 70., 1981.

Veihelmann, B., Nousiainen, T., Kahnert, M., and van der Zande, W. J.: Light scattering by small feldspar particles simulated using the Gaussian random sphere geometry, J. Quant. Spectrosc. Radiat. Transfer, 100, 393-405, 2006.

Vignati, E., Wilson, J., and Stier, P.: M7: an efficient size-resolved aerosol microphysics module for large-scale aerosol transport models, J. Geophys. Res., 109, D22202, doi:10.1029/2003JD004485, 2004.

Worringen, A., Ebert, M., Trautmann, T., Weinbruch, S., and Helas, G.: Optical properties of internally mixed ammonium sulfate and soot particles - a study of individual aerosol particles and ambient aerosol populations, Appl. Opt., 47, 3835-3845, 2008.

Xu, Y. and Gustafson, B. A. S.: A generalized multiparticle Miesolution: further experimental verification, J. Quant. Spectrosc. Radiat. Transfer, 70, 395-419, 2001.

Zhao, Y. and Ma, L.: Assessment of two fractal scattering models for the prediction of the optical characteristics of soot aggregates, J. Quant. Spectrosc. Radiat. Transfer, 110, 315-322, 2009. 